\title{
Sentiment Analysis Extensively to Harness Every Aspect of Primary School Education
}

\author{
Bhawna Tiwari (Ph.D. Research Scholar) \\ Department of Computer Science and Application \\ Sarvepalli Radhakrishnan University \\ NH 12,RKDF IST CAMPUS,HOSHANGABAD ROAD,MISROD,BHOPAL(M.P) \\ Dr.G.Santhi (Professor) \\ Department of Computer Science and Application \\ Sarvepalli Radhakrishnan University \\ NH 12,RKDF IST CAMPUS,HOSHANGABAD ROAD,MISROD,BHOPAL(M.P)
}

\begin{abstract}
The primary education sector is facing a significant transition to keep up to expectations in today's dynamic environment. Any part of primary school requires positive improvements. The paper highlights the importance of Opinion Analysis in the field of education in the Big Data era. With the generation of bulk data in recent years, innovations have been developed that make data collection and processing even simpler. A vast variety of data sets can be compiled, mined and made usable for sentiment analysis. Various target users, including the tutor, the pupil and the educational institution, have been discussed. The embodiment of human capital, tools and strategies, the education sector should make effective use of Sentiment Analysis to take account of all facets of primary school education. Thus, various algorithm are Naive Bayes, Complement Naive Bayes (CNB).
\end{abstract}

\section{INTRODUCTION}

Acquiring the most promising students has been a many challenge. Primary school has started to make comprehensive use of social media for marketing and promotional purposes. Parents, teachers and students and their families perform comprehensive internet study by mining data in order to achieve a deeper understanding of the prospective primary school. They communicate with students of common interest through blogs and other discussion sites and evaluate the standard of the colleges and universities involved. Personal considerations, education content, campus infrastructure, socialisation, financial support and policies are some of the main factors that students rely mainly on prior to enrolment. Research at the University of New Hampshire showed that 96 per cent of parents use Facebook, 84 per cent use YouTube, 20 per cent use blogs and 14 per cent use Twitter.

Application of Emotion Analysis methods would perform wonders if they were applied to the student enrolment process. The quality of every educational institution depends on the successful academic results and the student retention rate. The student's behaviour is an indicator of how well the student does academically. The student's overall college environment plays a vital role in academics. The implementation of cameras in school grounds offers an awareness of the student's behavioural behaviour in classrooms. , library, cafeteria, apartment, and so forth. The study of student databases relating to profile, archival activity and demography offers an intuitive diagnosis. Sentiment Analysis of social media data will effectively help universities frame curricula and improve the student retention rate. Big Data solutions have the necessary and effective approach for capturing, storing and analysing large datasets belonging to a number of data types and samples. Feedback research by student parents also 
plays a vital role for universities to have a homely environment in which students can be best shaped by making them feel at home. [5]. The framework stores student data such as grade and enrolment data and rankings to help students find the correct path in their academic life. Tableau, Quibble, Qlike is an example of several software that evaluate educational results. The remainder of the paper is structured as follows.

\section{Related work}

Sentiment research aims at emotional polarity, while many researcher are still recommend utilising a neutral category. M. Jiang et al[1] The neutral-class connection to the usefulness of classification was explored by contrasting twoclass models (positive/neutral, 3-class models (positive/neutral/neutral). You will find that twoclass models have indeed increased accuracy, but some scientists have performed best with 3-class models.

V. Solovyev et al[2] Includes mostly depressive feelings: writers use negatively validated words and prefer to discuss unfavourable sources. On the other hand, the discourse inside the series of social science textbooks written by Bogolubo has revealed a generally positive bias. The authors discuss the implications of these changes in relation to the future effects on studying the tone of the educational discourse.

L. Qiu, et al.[3] In order to further explore the love of affective functions, First of all, this paper explains the concept of Weibo and chooses smart campuses to research the influence of each function to feelings of the sentimental as the topic of Weibo's texts. These components are then fused into a multi-feature, and Tibetan microblog sentiment is classified as a multi-feature fusion. Experimental experiments have shown that the feature-fusion-based sentiment classification algorithm has increased the accuracy of the microblog sentiment classification.
F. Chen, et al[4] WS-MDL learns iteratively and selectively from low-cost and noise-containing emoticon labels on complex neural networks. Specifically, a probabilistic graphical model is used for the simultaneous acquisition of multimodal descriptors for philter mark noise and for the capture of modal dependence. Extensive research was carried out in the currency, real-world, Sina Weibo microblog sentiment dataset.

R. Liu, et al[5] Many scholars are expanding the transition from studying to the realm of emotional interpretation. This report reviews the research outcomes of sentiment analysis in Algorithms in recent years and for transfer learning and sentiment analysis applications, and we look forward to the progress of sentiment analysis.

W. Meng et al[6] Using BiLSTM to capture both local phrase characteristics and global and temporal phrase semantics. In addition, we are adding an attention function for modelling the relations between aspect terms and phrases in order to focus on some keywords of the objectives in order to learn more effectively about meaning representation. The proposed model is being tested on three datasets: Restaurant, Laptop and Twitter. Extensive trials have demonstrated the efficacy of FEA-NN.

L. Li et al[7] Given the linked characteristics of multiple social consumer identities and sequence, an efficient time+user double-consideration method model is proposed and implemented to interpret and compare public opinion's textual particulars. True data studies of the famous Chinese microblog forum, Sina Weibo, then test the effectiveness of the proposed model.

Y. Zhang, et al[8] Mark 2.214 Interactions through a range of independent platforms providing internet contact facilities. English is registered manually. ScenarioSa (2) describes how the two speakers linked to the relationships are related, and (3) reflects the sentimental development of each speaker through the conversation, which includes 
not only the sphere but also a broad variety of subjects and scenarios.

T. Zhou et al[9] Hen, a multimodal convolutionary neural network that could fully exploit crossmodality emotion interaction, is implemented and a better joint visual-textual representation is developed. The transfer learning methodology is more designed to reduce the effects of noise on real social data. Via extensive analyses on two datasets, we have shown that our proposed approach greatly exceeds the state-of-the-art methodology.the relationship between conventional visual-textual sentiment analysis and image regions. Finally, we suggest the expansion of interactive focus network models and the comparison of various ScenarioSA strong sentiment analysis algorithms, showing the need for novel models of interactive sentiment analysis.

\section{Proposed Methodology}

The proposed SA approach is intended to improve teaching and learning by encouraging the temporary and emotional analysis of multilingual student feedback in terms of teacher satisfaction and outcomes. The system categorises emotions in eight categories - anger, anticipation, disgust, fear, enthusiasm, disappointment, surprise and belief to be computed for happiness or unrest. Figure 1 show five main computer design components: data recovery, data preprocessing, feeling and emotional identification, pleasure and anger calculation, and data viewing. The system uses the open source R language (www.r-project.org) to do the pre-processing and emotional classification of the results.

Maximum Entropy The Maximum Entropy classifier is similar to the Naive Bayes classifier, except that instead of operating separately, the algorithm seeks weights for features that optimise the probability of search-based optimization training results. One of the drawbacks is that it does not make assurances on the relationship between characteristics, which is that, contrary to Naive
Bayes and SVM, it could work better if the requirements of conditional freedom are not fulfilled. One downside is that it is not really feasible in other issues, as individual datasets involve noise and error yielding some clean data[11]. For our high-entropy experiments, we use the $\mathrm{R}$ maxent package[4].

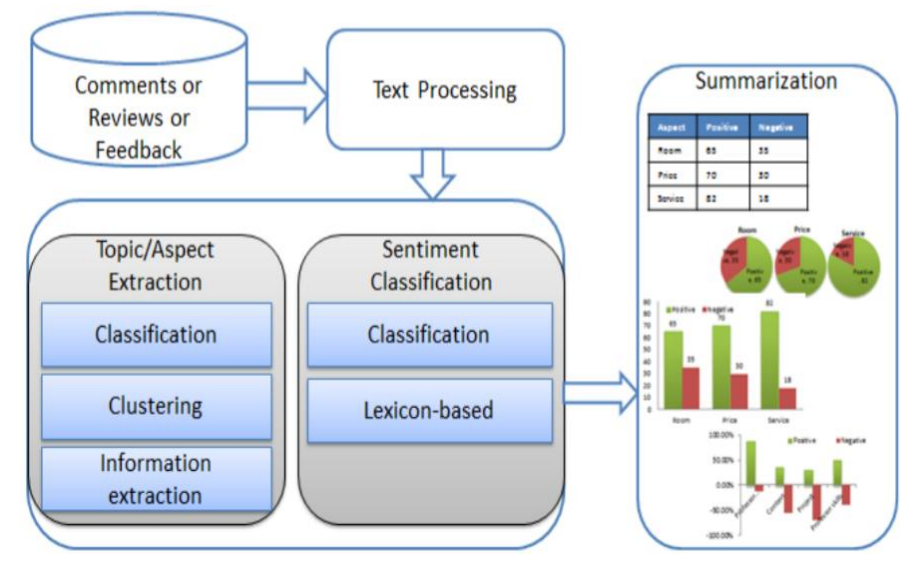

Figure 1: Sentiment Analysis Extensively To Harness Every Aspect Of Primary School Education

Support Vector Machines (SVM) The support vector machine is a modern kind of system of machine learning focused on the principle of statistical learning. The support vector machine has been the research subject of the machine learning world owing to excellent promotion and greater precision. This paper presents the basic theory of the support vector machine, the basic classification idea, and the classification method of the support vector machine commonly used. Practical issues with the algorithm, and evidence of the usefulness of the algorithm, the final outlook of the vector machine help prospects in classification applications. Finally, the possibility of assisting vector machines in classification applications is anticipated.

Experimental results indicate that the deletion of URLs barely affects the performance of the classifiers on all datasets in the two feature models. This means that URLs do not have useful information for the representation of emotions. The findings in Table 4 show that there is a need to delete the stop words for the description of emotions. Table 6 shows that the exclusion of 
numbers has no impact on the accuracy of the concept of emotion in the Priority Polarity Model, because the numbers are neutral. In the N-gram model, the deletion of numbers produces differences in the output of the classifier, with the exception of SVM. The productivity of SVM increases for all datasets after the number is deleted. The quality of SVM is improved following negation and the expansion of acronyms, thus allowing negation replacing and acronym extension in the SVM classification process. Returning words containing repeated letters will fluctuate production, so it's not advised to preprocess them. The random exclusion of the words results in a major decrease in the classification performance, since a missing key word will be the randomly excluded word, causing the decisionmaking polarity or damaging to the semantine ratio of the penalty. Experimental results reveal that the same pre-processing approach is usually brief with comparatively simple emotional speech and simple grammar and that texts with common grammatical features share common related expressions of emotions. This section classifies emotions by examining the emotional relations to the text sequences the nostalgic characteristics, The characteristics of the possible sequence are extracted from the preparation corpus, followed by a minimum assist function and a minimum conviction as a vector unit. Second, the series is vectorized according to vector characteristics, the classification model is defined and the classification of the microblog is checked.

A basic sequence mixture is more common in the microblog corpus, but there are also some unusual sequence variations, so a sequence function can't be chosen only with restricted help and minimal trust. We suggest a method of multi minimum to address this problem, where the minimum support for the law Depends on the limited artefact product occurrence in the training set and parameter. Not only does this technique maintain uncommon sequence features with low funding, it can overcome over-fitting with regular sequence features as well. A microblog sensation process sequence features based and show in figure The specific stages of microblog sentiment grouping dependent on sequence features are:

1. Serialize the instructional Corpus on the grounds of the properties of emotional terms, degrading words and conjunctures. If the text has a conjunct feature, it would be used as a single entity.

2. Build an n-dimensional function vector based on a sequence mining pattern based on a multiminimal support strategy.

3. The corpus to be classified is represented using classification models that are educated in the corpus.

\section{Conclusion}

Data mining tools and techniques have a major role play in the exploration of valuable high-quality information buried in vast quantities of data on the Internet. It could be discovered by adequate filtering, exploitation and analysis. Applying data mining to social media content, known as social media analytics allows companies to analyse the data they have gathered from social media platforms and to consider their customers in depth, including their interests, feelings and views. Study in sentiment analysis has demonstrated that social media is an outstanding platform for finding habits and trends and for greater comprehension of consumer attitudes and desires, such that goods or services can be proactively innovated. Experiences from other areas, as well as some of the sources listed in the article, indicate that sentiment analysis may also be useful for higher education. The analysis of social media content created by students in higher education could provide valuable insight into their attitudes, opinions and feelings towards schools, curricula, topics, student relations, support offered by non-teachers, and other areas of research. 


\section{Reference}

[1]. M. Jiang, J. Wu, X. Shi and M. Zhang, "Transformer Based Memory Network for Sentiment Analysis of Web Comments," in IEEE Access, vol. 7, pp. 179942-179953, 2019, doi: 10.1109/ACCESS.2019.2957192.

[2]. V. Solovyev, M. Solnyshkina, E. Gafiyatova, D. McNamara and V. Ivanov, "Sentiment in Academic Texts," 2019 24th Conference of Open Innovations Association (FRUCT), Moscow, Russia, 2019, pp. 408-414, doi: 10.23919/FRUCT.2019.8711900.

[3]. L. Qiu, Q. Lei and Z. Zhang, "Advanced Sentiment Classification of Tibetan Microblogs on Smart Campuses Based on Multi-Feature Fusion," in IEEE Access, vol. 6, pp. 17896-17904, 2018, doi: 10.1109/ACCESS.2018.2820163.

[4]. F. Chen, R. Ji, J. Su, D. Cao and Y. Gao, "Predicting Microblog Sentiments via Weakly Supervised Multimodal Deep Learning," in IEEE Transactions on Multimedia, vol. 20, no. 4, pp. 997-1007, April 2018, doi: 10.1109/TMM.2017.2757769.

[5]. R. Liu, Y. Shi, C. Ji and M. Jia, "A Survey of Sentiment Analysis Based on Transfer Learning," in IEEE Access, vol. 7, pp. 8540185412, 2019, doi: 10.1109/ACCESS.2019.2925059.

[6]. W. Meng, Y. Wei, P. Liu, Z. Zhu and H. Yin, "Aspect Based Sentiment Analysis With Feature Enhanced Attention CNN-BiLSTM," in IEEE Access, vol. 7, pp. 167240-167249, 2019, doi: 10.1109/ACCESS.2019.2952888.

[7]. L. Li, Y. Wu, Y. Zhang and T. Zhao, "Time+User Dual Attention Based Sentiment Prediction for Multiple Social Network Texts With Time Series," in IEEE Access, vol. 7, pp. 17644-17653, 2019, doi: 10.1109/ACCESS.2019.2895897.

[8]. Y. Zhang, Z. Zhao, P. Wang, X. Li, L. Rong and D. Song, "ScenarioSA: A Dyadic Conversational Database for Interactive Sentiment Analysis," in IEEE Access, vol. 8, pp. 90652-90664, 2020, doi: 10.1109/ACCESS.2020.2994147.
[9]. T. Zhou, J. Cao, X. Zhu, B. Liu and S. Li, "Visual-Textual Sentiment Analysis Enhanced by Hierarchical Cross-Modality Interaction," in IEEE Systems Journal, doi: 10.1109/JSYST.2020.3026879.

[10]. H. Zhang et al., "Multidimensional Extra Evidence Mining for Image Sentiment Analysis," in IEEE Access, vol. 8, pp. 103619-103634, 2020, doi: 10.1109/ACCESS.2020.2999128.

[11]. W. Zheng, Z. Xu, Y. Rao, H. Xie, F. L. Wang and R. Kwan, "Sentiment classification of short text using sentimental context," 2017 International Conference on Behavioral, Economic, Socio-cultural Computing (BESC), Krakow, 2017, pp. 1-6, doi: 10.1109/BESC.2017.8256405.

[12]. B. Liu et al., "Context-aware social media user sentiment analysis," in Tsinghua Science and Technology, vol. 25, no. 4, pp. 528-541, Aug. 2020, doi: 10.26599/TST.2019.9010021.

[13]. R. Xia, F. Xu, C. Zong, Q. Li, Y. Qi and T. Li, "Dual Sentiment Analysis: Considering Two Sides of One Review," in IEEE Transactions on Knowledge and Data Engineering, vol. 27, no. 8, pp. 2120-2133, 1 Aug. 2015, doi: 10.1109/TKDE.2015.2407371.

[14]. M. R. Yaakub, F. Z. M. Zaki, M. I. A. Latiffi and S. Danby, "Sentiment analysis of preschool teachers' perceptions on ICT use for young children," 2019 IEEE International Conference on Engineering, Technology and Education (TALE), Yogyakarta, Indonesia, 2019, pp. 1-6, doi: 10.1109/TALE48000.2019.9225938.

[15]. Y. Gao, J. Liu, P. Li and D. Zhou, "CEHEAT: An Aspect-Level Sentiment Classification Approach With Collaborative Extraction Hierarchical Attention Network," in IEEE Access, vol. 7, pp. 168548-168556, 2019, doi: 10.1109/ACCESS.2019.2954590.

[16]. F. F. Balahadia, M. C. G. Fernando and I. C. Juanatas, "Teacher's performance evaluation tool using opinion mining with sentiment analysis," 2016 IEEE Region 10 Symposium (TENSYMP), Bali, 2016, pp. 95-98, doi: 10.1109/TENCONSpring.2016.7519384. 
[17]. N. Anggraini and M. J. Tursina, "Sentiment Analysis of School Zoning System On Youtube Social Media Using The K-Nearest Neighbor With Levenshtein Distance Algorithm," $2019 \quad$ 7th International Conference on Cyber and IT Service Management (CITSM), Jakarta, Indonesia, 2019, pp. 1-4, doi: 10.1109/CITSM47753.2019.8965407.

[18]. NITIN, Gokran Ila; GOTTIPATI Swapna; and SHANKARARAMAN, Venky. Analyzing Educational Comments for Topics and Sentiments: A Text Analytics Approach. (2015). Frontiers in Education Conference 2015: Proceedings: El Paso, Texas, October 21-24. 1658-1666. Research Collection School Of Information Systems. 